\title{
Salvage treatment after radical prostatectomy
}

Salvage radiotherapy to the prostate bed is a standard approach for men with PSA failure after radical prostatectomy. It achieves biochemical control in around half of cases, and is thought to improve overall survival [1]. The addition of hormone therapy to radiation enhances the efficacy of primary treatment [2], but until now, it was not clear whether it also improved the outcome of salvage radiation.

In this month's issue of European Urology, Spratt et al have reviewed the two randomized trials addressing this issue [3]. They rightly focus more on the RTOG 9601 trial [4] because the longer follow-up provides more data on clinically meaningful long-term outcomes. The results clearly prove the principle that the addition of hormone therapy can improve clinical outcomes after salvage prostate bed radiotherapy. Among men receiving radiation alone, one half of the deaths so far were from prostate cancer (64/131). Among those receiving bicalutamide in addition, one third of the deaths so far were from prostate cancer $(34 / 108)$. The point estimate for the absolute benefit of two years of adjuvant bicalutamide was 5\% in overall survival, and $8 \%$ in cause-specific survival. Given that bicalutamide is both cheap and well tolerated, this is a substantial benefit. Treating just 13 men with bicalutamide to prevent one of them dying from prostate cancer is worthwhile.

Spratt et al helpfully address the need to identify which patient groups may benefit most from adjuvant bicalutamide. They conclude 
that "men who benefit most ... are those with a ... PSA $>=0.7 \mathrm{ng} / \mathrm{ml}$, positive margins and Gleason 8-10". This conclusion is open to some misinterpretation because "with the exception of PSA level, interaction tests did not indicate a significant differential benefit" [4]. In other words, the data from RTOG 9601 are consistent with the same relative survival benefit regardless of margin status or Gleason score. Of course, for a given relative benefit, patients with a worse prognosis will gain a greater absolute benefit.

The potential predictive role of post-operative, pre-radiotherapy PSA level is less clear because, as Shipley et al indicate [4], there was a statistically significant interaction of PSA with the treatment effect. However, even this apparent subgroup effect should not be accepted uncritically. It is perhaps surprising, but nonetheless true, that most subgroup effects, even those with a significant test for interaction, are not reproducible [5].

When a clinical trial reports a potential subgroup effect there are standard criteria to judge the credibility of that claim [6]. In this case, there are several factors casting doubt on the reliability of the apparent interaction between PSA level and reported benefit from adjuvant bicalutamide. The subgroup analysis, the PSA cut-points used, and the direction of the effect, were not pre-specified; the effect was not consistent across other outcome measures such as freedom from metastases; validation from other studies is lacking; and there is no obvious biological rationale. In our view, the observation of an interaction between PSA level and treatment effect should be 
regarded as hypothesis generating at this time, rather than practice changing.

How should men with PSA failure after radical prostatectomy be managed in the light of current evidence? The most important predictors of overall survival in RTOG 9601 were age and Gleason score. For older men with a shorter life expectancy, particularly those with a lower Gleason score, salvage radiation alone is a reasonable option. For healthier younger men, particularly those with a higher Gleason score, there is a strong case for adjuvant hormone therapy in addition. Given the evidence that LHRH agonists are more effective than bicalutamide monotherapy in other settings [7], we favour the former. The optimum duration of LHRH agonists when used in this setting is unknown: one randomised comparison from the RADICALS-HD trial is a comparison of 6 months versus 24 months LHRH agonists [8]. This includes 880 patients having RT in the early salvage setting, and 643 patients having RT in the adjuvant setting.

There are several important questions for further research. Treatment efficacy might be improved further by the use of pelvic nodal irradiation in addition to treatment of the prostate bed, as the authors note [3], or by the use of newer Androgen Receptor-targeted therapy. Furthermore, as imaging techniques improve, it is possible that salvage treatment could be safely delayed until the site of recurrent disease is detectable. Those men with disseminated disease could then avoid the need for local radiotherapy, whereas for 
those with localized or oligometastatic disease, radiotherapy could be appropriately tailored to each individual.

\section{References}

[1] Tendulkar RD, Agrawal S, Gao T et al. , Contemporary Update of a Multi-Institutional Predictive Nomogram for Salvage Radiotherapy After Radical Prostatectomy. J Clin Oncol. 2016 Aug 15. [Epub ahead of print]

[2] Kumar S , Shelley M, Harrison C et al. Neo-adjuvant and adjuvant hormone therapy for localised and locally advanced prostate cancer. Cochrane Database Syst Rev. 2006 Oct $18 ;(4)$

[3] Spratt DE, Dess RT, Zumsteg ZS et al. A Systematic Review and Framework for the Use of Hormone Therapy with Salvage Radiation Therapy for Recurrent Prostate Cancer. European Urology (in press).

[4] Shipley WU, Seiferheld W, Lukka HR et al. Radiation with or without Antiandrogen Therapy in Recurrent Prostate Cancer. N Engl J Med. 2017 Feb 2;376(5):417-428.

[5] Wallach JD, Sullivan PG, Trepanowski JF et al. Evaluation of Evidence of Statistical Support and Corroboration of Subgroup 
Claims in Randomized Clinical Trials. JAMA Intern Med. 2017 Apr $1 ; 177(4): 554-560$

[6] Sun X, Briel M, Busse JW et al. Credibility of claims of subgroup effects in randomised controlled trials: systematic review. BMJ. 2012 Mar 15;344:e1553

[7] Tyrrell CJ, Kaisary AV, Iversen P et al. A randomised comparison of 'Casodex' (bicalutamide) $150 \mathrm{mg}$ monotherapy versus castration in the treatment of metastatic and locally advanced prostate cancer. Eur Urol. 1998; 33(5):447-56.

[8] Parker C, Sydes MR, Catton C et al. Radiotherapy and androgen deprivation in combination after local surgery (RADICALS): a new Medical Research Council/National Cancer Institute of Canada phase III trial of adjuvant treatment after radical prostatectomy. BJU Int. 2007 Jun;99(6):1376-9. 